\title{
Somatosympathetic vasoconstrictor reflexes in human spinal cord injury: responses to innocuous and noxious sensory stimulation below lesion
}

\author{
Vaughan G. Macefield *, Alexander R. Burton and Rachael Brown \\ School of Medicine, University of Western Sydney and Neuroscience Research Australia, Sydney, NSW, Australia
}

\section{Edited by:}

Alexander Rabchevsky, University of

Kentucky, USA

Reviewed by:

David C. Randall, University of Kentucky College of Medicine, USA

Andrei Krassioukov, International

Collaboration on Repair Discoveries,

Canada

\section{*Correspondence:}

Vaughan G. Macefield, School of Medicine, University of Western Sydney, Locked Bag 1797, Penrith, Sydney, NSW 2751, Australia.

e-mail:v.macefield@uws.edu.au
It is known that the sudden increases in blood pressure associated with autonomic dysreflexia in people with spinal cord injury (SCI) are due to a spinally mediated reflex activation of sympathetic vasoconstrictor neurons supplying skeletal muscle and the gut. Apart from visceral inputs, such as those originating from a distended bladder, there is a prevailing opinion that autonomic dysreflexia can be triggered by noxious stimulation below the lesion. However, do noxious inputs really cause an increase in blood pressure in $\mathrm{SCl}$ ? Using microelectrodes inserted into a peripheral nerve to record sympathetic nerve activity we had previously shown that selective stimulation of small-diameter afferents in muscle or skin, induced by bolus injection of hypertonic saline into the tibialis anterior muscle or the overlying skin, evokes a sustained increase in muscle sympathetic nerve activity and blood pressure and a transient increase in skin sympathetic nerve activity and decrease in skin blood flow in able-bodied subjects. We postulated that these sympathetic responses would be exaggerated in $\mathrm{SCl}$, with a purely noxious stimulus causing long-lasting increases in blood pressure and long-lasting decreases in skin blood flow. Surprisingly, though, we found that intramuscular or subcutaneous injection of hypertonic saline into the leg caused negligible changes in these parameters. Conversely, weak electrical stimulation over the abdominal wall, which in able-bodied subjects is not painful and activates large-diameter cutaneous afferents, caused a marked increase in blood pressure in $\mathrm{SCl}$ but not in ablebodied subjects. This suggests that it is activation of large-diameter somatic afferents, not small-diameter afferents, that triggers increases in sympathetic outflow in SCl. Whether the responses to activation of large-diameter afferents reflect plastic changes in the spinal cord in $\mathrm{SCl}$ is unknown.

Keywords: autonomic dysreflexia, innocuous stimulation, noxious stimulation, spinal cord injury, sympathetic nervous system

\section{INTRODUCTION}

While spinal cord injury (SCI) can cause devastating changes in the somatic nervous system below the lesion - paralysis and loss of sensation - compared to our knowledge of the sensory and motor disturbances following a spinal injury relatively little is known about changes to the sympathetic nervous system (McLachlan and Brock, 2006). Given that the sympathetic supply to the viscera and blood vessels is derived from the thoracic and lumbar segments of the spinal cord, an injury to the cord can - depending on the level and extent of the lesion - result in partial or complete loss of descending control of sympathetic function below the lesion. With high thoracic or cervical lesions the loss of sympathetic vasoconstrictor drive to blood vessels in muscles and the gut results in a low resting blood pressure and orthostatic hypotension; control of skin blood flow, and sweat release, and hence thermoregulatory control, is also compromised (Mathias and Frankel, 2002). However, because segmental spinal circuitry is intact, sensory stimuli originating below the lesion can cause a spinally mediated reflex activation of sympathetic vasoconstrictor neurons and a consequent increase in blood pressure. While it is known that visceral inputs, such as those from the bladder or bowel, can trigger these reflex vasoconstrictor responses, it is generally believed that non-visceral (somatic) inputs must be in the noxious range in order to generate similar vasoconstrictor responses. The purpose of this review is to contextualize recent experimental evidence that addresses the dogma surrounding the types of somatic inputs involved in the generation of spinal somatosympathetic reflexes.

\section{AUTONOMIC DYSREFLEXIA}

Following SCI reflex activation of sympathetic preganglionic vasoconstrictor neurons by sensory stimuli below lesion can cause arterial pressure to rise so suddenly and remain at dangerously high levels - leading to retinal and subarachnoid hemorrhage, stroke, and (occasionally) cardiac arrest - that this phenomenon, termed autonomic dysreflexia, is considered a medical emergency (Karlsson, 1999, 2006; Mathias and Frankel, 2002). It is common for midthoracic lesions and above ( $>$ T6, i.e., above the level of sympathetic outflow to the splanchnic circulation), where many vascular beds may have been decentralized and the only means available to offset the hypertension is to slow down the heart via 
the vagus nerve (which, because it exits from the base of the skull, is spared in spinal cord injuries). Moreover, denervation supersensitivity of the vessels has been documented in human SCI, such that vascular responses to noradrenaline are greatly exaggerated (Mathias et al., 1976; Arnold et al., 1995), and work in spinalized rats has shown that neurovascular transmission is potentiated (Brock et al., 2006; Rummery et al., 2010).

Although autonomic dysreflexia has been reported in low thoracic (T8-T10) lesions (Gimovski et al., 1985), usually enough vascular beds are under supraspinal control that adequate vasodilatation can be brought about via the normal baroreceptor-mediated withdrawal of vasoconstrictor drive (Teasell et al., 2000). Autonomic dysreflexia is defined as an increase in systolic BP greater than $20 \mathrm{mmHg}$ above baseline, combined with one or more subjective or objective clinical symptoms (Karlsson, 1999). The typical subjective signs of autonomic dysreflexia include a throbbing headache, tingling in the head or nasal congestion; sweating and flushing above the lesion, and (if observed) pupillary dilation, are clinical signs that prompt medical staff to measure blood pressure and to locate the source of sensory stimulation (usually a distended bladder or impacted colon, sometimes - according to clinical doctrine - a pressure sore or ingrown toenail). However, there is no correlation between the magnitude of the hypertensive response and the headache (Teasell et al., 2000). Moreover, by definition, subclinical episodes go undetected, and this syndrome of silent dysreflexia is of increasing concern, particularly for those living with SCI in the community (Linsenmeyer et al., 1996).

\section{ASSESSING SYMPATHETIC OUTFLOW BELOW LESION FOLLOWING HUMAN SPINAL CORD INJURY}

In intact individuals sympathetic control of blood vessels in muscle is specific to the maintenance of blood pressure, while sympathetic outflow to the skin primarily subserves thermoregulation through its actions on cutaneous blood vessels, sweat glands, and (phylogenetically) hairs. Direct recordings of muscle sympathetic nerve activity (MSNA), which are made routinely in our laboratory via tungsten microelectrodes inserted percutaneously into a peripheral nerve (microneurography), reveal ongoing bursts of MSNA that are phase-locked to the cardiac cycle via the baroreflex and which increase during maneuvres that unload the baroreceptors (Delius et al., 1972a). Conversely, bursts of skin sympathetic nerve activity (SSNA) are largely unrelated to the cardiac cycle but increase during whole-body cooling (which causes an increase in cutaneous vasoconstrictor drive) or whole-body heating (which brings about an increase in sudomotor drive); bursts of SSNA can also be evoked by arousal stimuli, such as unexpected loud sounds or electrical stimuli to the skin, and the overall level of SSNA is elevated during states of anxiety (Delius et al., 1972b). In patients with complete high thoracic or cervical spinal lesions there is essentially no spontaneous MSNA or SSNA below the lesion because the descending supraspinal excitatory drive to the preganglionic neurons - located in the thoracolumbar spinal cord - is interrupted, and there are no spontaneous fluctuations in skin blood flow or sweat release below the lesion (Wallin and Stjernberg, 1984; Stjernberg et al., 1986). Moreover, the differential control of MSNA and SSNA seen in intact individuals is lost: vasoconstrictor neurons supplying muscle and skin, as well as sudomotor and pilomotor neurons, are coactivated by visceral or somatic sensory inputs originating below the level of the lesion (Wallin and Stjernberg, 1984; Stjernberg et al., 1986). In intact individuals, distension of the bladder - caused by drinking water - causes an increase in both the amplitude and frequency of spontaneous bursts of MSNA and increases in systolic and diastolic pressure (Fagius and Karhuvaara, 1989). Although the same experiment has not been done following SCI, firm pushing over the lower abdomen to compress the bladder wall causes a very marked increase in blood pressure yet only brief bursts of MSNA that are modest in amplitude (Wallin and Stjernberg, 1984; Stjernberg et al., 1986). Despite this relatively weak sympathetic neural response, repeated bouts of bladder percussion cause a large increase in noradrenaline spillover below the lesion (Karlsson et al., 1998). This suggests that a given increase in sympathetic neural traffic causes a greatly increased release of noradrenaline (and co-transmitters) from the sympathetic terminals.

Given the importance of the sympathetic nervous system in the genesis of autonomic dysreflexia, it is surprising that assessment of injury in terms of autonomic involvement is still lacking in patients with SCI. Moreover, unlike studies of spinal injury in experimental animals - in which lesions are often complete transactions performed surgically - the diverse nature of accidents precipitating human SCI means that the lesions are usually incomplete. In addition, many individuals regain some functional recovery over time. Accordingly, just as it is important to know the status of the somatosensory and somatomotor nervous systems below a spinal lesion, it is equally important to know the status of the sympathetic nervous system. In 2009, an Autonomic Standards Assessment Form was released to document the impact of SCI on autonomic function (Alexander et al., 2009). However, this assessment tool does not give an autonomic level of injury, rather a framework for the assessment of specific autonomic functions following SCI. Level of injury is still based solely on motor and sensory function and is defined neurologically as the most caudal segment at which both motor and sensory functions are intact. The American Spinal Injury Association (ASIA) impairment scale (AIS; Marino et al., 2003) provides a score as to whether the injury is complete or incomplete, with a complete injury (ASIA A) being defined as complete loss of motor or sensory function in sacral segments S4-S5. An incomplete injury is classified as ASIA B, C, or $\mathrm{D}$ depending upon the degree of preservation of motor and sensory function. Various approaches have been used to assess the integrity of sympathetic pathways through and below a lesion in human SCI.

While microneurography can record sympathetic neural activity directly, it is a very time-consuming procedure to perform in the clinical setting and is not typically used for routine clinical diagnosis. Moreover, microneurography can be very difficult to undertake in individuals with SCI because of the decreased excitability of motor nerves, which makes finding muscle fascicles of the nerve difficult (Lin et al., 2007). Nevertheless, recording the effector-organ responses does provide a means of assessing sympathetic function indirectly: an increase in muscle and/or splanchnic vasoconstrictor drive can be inferred from an increase in blood pressure (assuming heart rate does not increase in parallel), while an increase in cutaneous vasoconstrictor or sudomotor drive can 
be inferred by a decrease in skin blood flow (measured by laser Doppler or photoelectric plethysmography) or by an increase in sweat release (measured indirectly from changes in electrical resistance, conductance, or potential of the skin - termed the galvanic skin response or the skin sympathetic response). Indeed, there have been several attempts to assess the autonomic completeness of a spinal lesion by recording the electrodermal responses to electrical stimulation below and above lesion (Fuhrer, 1975; Cariga et al., 2002; Reitz et al., 2003; Ogura et al., 2004). While each of these studies found that a complete spinal lesion abolishes sudomotor responses in the lower limbs to electrical stimulation above lesion, Cariga et al. (2002) concluded that the isolated spinal cord cannot generate cutaneous sympathetic responses, as demonstrated by the absence of electrodermal responses in the lower limbs to stimuli delivered below lesion. While sudomotor responses to electrical stimulation below the lesion have occasionally been found (Fuhrer, 1975; Reitz et al., 2003), in our experience sudomotor responses in SCI are very unreliable markers of increases in cutaneous sympathetic outflow (Brown et al., 2007, 2009a,b; Brown and Macefield, 2008).

Recording of skin vasomotor responses below lesion to arousal stimuli has been suggested as a means of assessing the integrity of sympathetic pathways (Nicotra et al., 2005), and earlier studies had shown that skin vasoconstrictor responses can be elicited by sensory stimuli delivered below lesion (Wallin and Stjernberg, 1984; Stjernberg et al., 1986; Karlsson et al., 1998). Indeed, we recently showed that cutaneous vasomotor responses to stimuli delivered above and below lesion provide robust markers as to the integrity of cutaneous sympathetic pathways both through and below a spinal lesion (Brown et al., 2007, 2009a,b; Brown and Macefield, 2008). We have also used the blood pressure and heart rate responses to a maximal inspiratory breath-hold as a robust indicator of the integrity of muscle sympathetic outflow in SCI: in spinal injury this maneuvre causes a sustained fall in blood pressure and a compensatory increase in heart rate, the magnitudes of which depend on the level of the lesion, whereas in intact individuals the sustained increase in MSNA causes diastolic pressure to return to normal levels after an initial fall; heart rate remains stable (Brown and Macefield, 2008).

\section{SOMATOSYMPATHETIC REFLEXES IN EXPERIMENTAL ANIMALS}

The increase in blood pressure from sensory stimulation of the body wall is an example of a somatosympathetic reflex, while that to distension of the bladder or rectum is an example of a viscero sympathetic reflex. As noted above, while visceral inputs are known to be potent triggers of autonomic dysreflexia, it is generally assumed that any somatic stimuli must be noxious in order to induce significant increases in blood pressure. Indeed, the term "somatosympathetic reflexes" has been used specifically to refer to the autonomic responses to noxious stimuli originating below lesion (Saper and DeMarchena, 1986). However, it is known that light stroking of the skin is capable of inducing autonomic dysreflexia in spinalized rats, as is colonic distension at pressure levels lower than those that evoke signs of pain in intact rats (Marsh and Weaver, 2004), arguing against the idea that noxious stimulation is a requirement for evoking somatosympathetic reflexes in SCI. Somatosympathetic reflexes have been well characterized in the cat and rat, and have been shown to comprise both spinal and supraspinal components. Electrical stimulation of peripheral nerves or dorsal roots induces a biphasic response comprising a short-latency and a long-latency component: the latter depends on supraspinal circuitry, whereas the short-latency component is a spinal reflex that is arranged segmentally (Coote and Downman, 1966; Coote et al., 1969; Sato and Schmidt, 1971, 1973; Kerman and Yates, 1999). It has been shown that electrical stimulation of thoracic dorsal roots induces reflex activation of sympathetic preganglionic neurons with stimulation frequencies of up to $20 \mathrm{~Hz}$, the amplitude of the responses decreasing at higher frequencies (Beacham and Perl, 1964). For some preganglionic neurons single afferent stimuli could induce reflex responses after an interval of only $12 \mathrm{~ms}$, but tetanic stimulation of the afferents did not lead to augmented responses (Beacham and Perl, 1964). The afferent axons are believed to be myelinated, given that long-latency reflex responses can be evoked at stimulation intensities of $1.5 \times$ sensory (dorsal root) threshold (T), and short-latency components at 5T (Sato and Schmidt, 1971), yet most studies have used maximal stimuli (e.g., 50T) - intensities that also activate unmyelinated (C) fibers. Indeed, studies of somatosympathetic reflexes in the spinal cat have mostly used intensities of electrical stimulation that were within the noxious range, thereby activating both myelinated and unmyelinated afferents (Horeyseck and Jänig, 1974; Jänig and Spilok, 1978). Apart from these early studies on the relationships between the intensity of the somatic inputs and the sympathetic responses, little is known of the input-output relationships for somatosympathetic reflexes, although recent work has begun to redress this (McMullan et al., 2008). However, even less is known about these reflexes in humans.

\section{SOMATOSYMPATHETIC REFLEXES TO NON-NOXIOUS INPUTS IN HUMAN SPINAL CORD INJURY}

By definition, an increase in sympathetic outflow in response to sensory inputs originating below the level of a complete spinal lesion is spinally mediated, so human SCI provides a clinical model in which to examine spinal somatosympathetic reflexes. Interruption of the descending vasomotor pathways by a spinal lesion leaves the spinal sympathetic preganglionic neurons below the lesion deprived of supraspinal control, yet open to sensory inputs from below the lesion. We had previously shown (Brown et al., 2007) that by delivering brief $(1 \mathrm{~s})$ innocuous trains of electrical stimuli (3-10 mA, $0.2 \mathrm{~ms}$ pulses @ 20 Hz) to the forehead at unexpected times we could evoke sympathetically mediated cutaneous vasoconstrictor and sudomotor responses. These are simply arousal responses, but if we can record these from the toes in a patient with a high lesion this indicates that descending sympathetic pathways are intact.

Experimental records from a patient with a neurologically complete (ASIA A) cervical lesion (C6), sustained 4 months previously, are shown in Figure 1. Although not shown, electrical stimulation over the forehead failed to elicit cutaneous vasoconstriction (or sweat release) in the fingers and toes, indicating that descending sympathetic pathways were effectively interrupted. Conversely, electrical stimuli delivered to the abdominal wall (i.e., below the lesion) did evoke vasoconstriction in the fingers and toes. In this 
C6 ASIA A +4 months

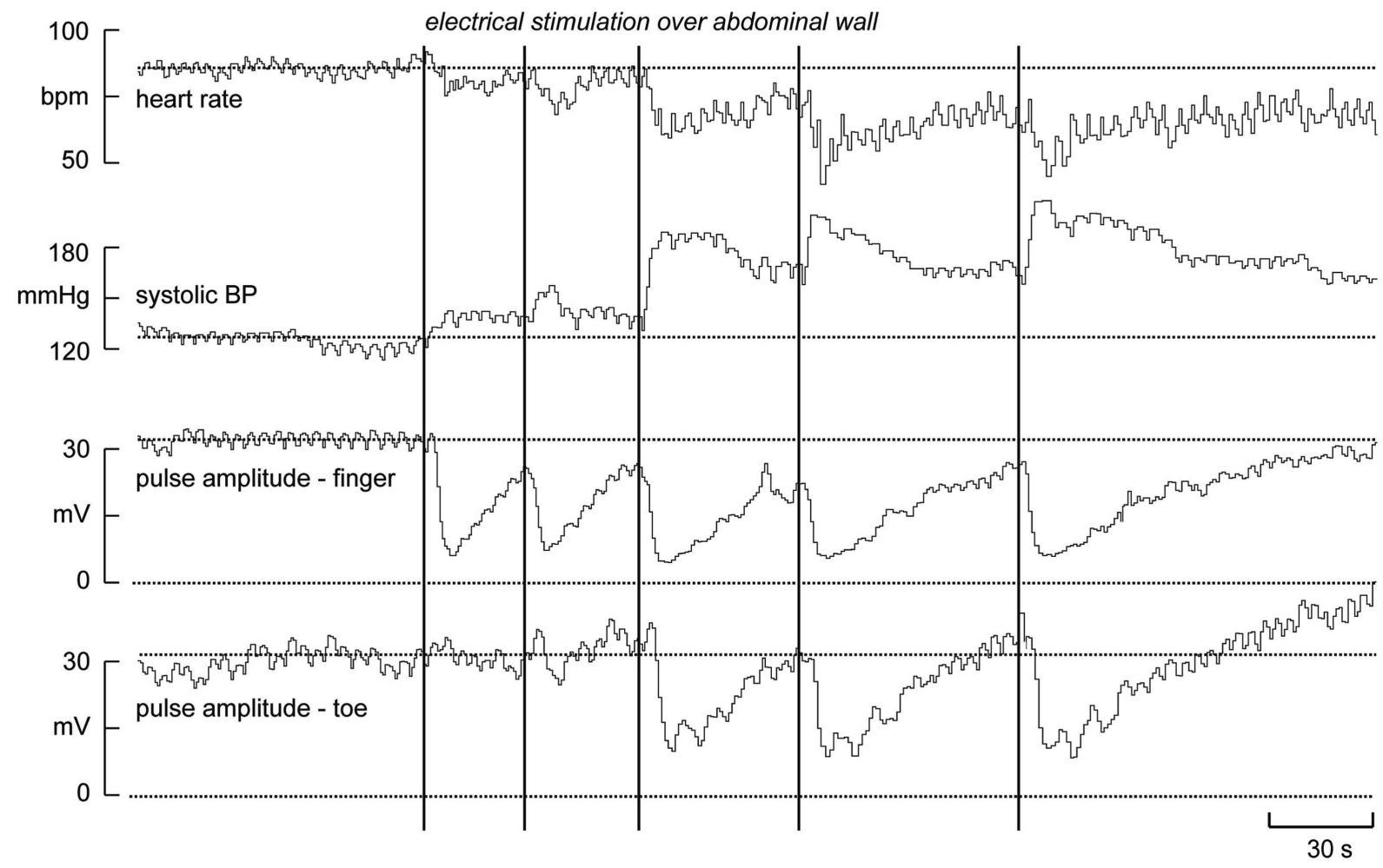

FIGURE 1 | Heart rate, systolic pressure, and skin blood volume recorded from a finger and toe in a patient who had sustained a $\mathbf{C 6}$ lesion.

Electrical stimuli (vertical lines) applied to the abdominal wall (i.e., below the lesion) caused vasoconstriction in both the fingers and toes, an increase in systolic pressure and a baroreflex-mediated bradycardia (modified from Brown et al., 2007). example we found evidence of "build-up," in which the effectiveness of the vasoconstrictor outflow increases with subsequent stimuli. This phenomenon could, perhaps, be seen as evidence of short-term plasticity in the spinal circuitry, though it might reflect changes at the level of neurovascular transfer. The first two stimuli applied to the abdominal wall - which in intact individuals were considered surprising but not noxious - caused no vasoconstriction in the toes and, while marked vasoconstriction was observed in the fingers, the stimuli caused negligible increases in arterial pressure. However, when vasomotor responses were observed in the toes (with the third stimulus) a significant increase in systolic pressure was generated. Presumably, this reflects constriction in a larger area of the vascular beds below the lesion. Moreover, it can be seen that the hypertensive response, and the resultant vagally mediated bradycardia, is cumulative. Clearly, because the subject could not feel the stimuli these vasoconstrictor responses represent spinal somatosympathetic reflexes rather than arousal responses. Unlike reductions in skin blood flow, sweat release - another marker of cutaneous sympathetic outflow - was rarely observed below the lesion. Indeed, sudomotor responses to abdominal stimulation could not be detected in the hands and feet in any of the patients with lesions above T7 (Brown et al., 2007).

In another study we examined the input-output relationships of the vasoconstrictor responses induced by electrical stimulation of the abdominal wall, testing the hypothesis that the magnitude and duration of the vasoconstriction was directly related to the duration of the stimulus. We predicted that a $20 \mathrm{~s}$ train of electrical stimuli would cause a longer period of vasoconstriction than that caused by a $1 \mathrm{~s}$ train of identical frequency, and that a train of 20 pulses delivered over 20 s (i.e., a stimulation frequency of $1 \mathrm{~Hz}$ ) would cause a smaller vasoconstrictor response than that caused by delivering the 20 pulses over $1 \mathrm{~s}$ (stimulation frequency $20 \mathrm{~Hz}$ ). Interestingly, we showed that the magnitude and duration of the somatosympathetic reflex response to electrical stimulation applied below lesion is not related to the duration and intensity of the sensory input; there was no difference in magnitude or duration of cutaneous vasoconstriction below the lesion for each of the stimulus parameters, suggesting that the recruitment of cutaneous (and muscle and splanchnic) vasoconstrictor neurons may be limited to the initial afferent barrage evoked by a stimulus train (Brown et al., 2009a). However, we do not know whether this is due to habituation of the spinal somatosympathetic reflex with longer trains or represents changes in neurotransmitter release and/or changes in vascular reactivity.

As noted above, Cariga et al. (2002) found that they could not evoke sudomotor responses in any patients during stimulation of peripheral nerves below the lesion. While we agree that sudomotor responses can only rarely be obtained, our demonstration of robust vasoconstrictor responses in the skin (and, by inference, other vascular beds) does not support the conclusion made 
by these authors that the isolated spinal cord is unable to generate cutaneous sympathetic responses. Unlike the poor sudomotor responses, assessment of cutaneous vasomotor responses provides a very robust measure of the state of the sympathetic nervous system below lesion; moreover, one can plot the time course of the vasoconstrictor response by measuring the fall in cutaneous perfusion with every heart beat; this is a proxy marker of skin blood flow. We measured sudomotor and vasomotor responses, together with continuous blood pressure and heart rate, in another study from our laboratory that specifically targeted autonomic dysreflexia using non-noxious stimulation in a clinical setting. In six quadriplegic (C3-C7) and four paraplegic (T3-T6) men, vibroejaculation - brought about by vibration of the penis - was performed as a means of assessing fertility (Brown et al., 2009b). This is an inherently dangerous procedure, given the risks of triggering autonomic dysreflexia (Brackett et al., 1998); that the dysreflexia is abolished by anesthetic block of the dorsal penile nerve indicates that the increase in blood pressure is brought about by a somatosympathetic reflex (Wieder et al., 2000). In our study, vibration of the penis caused systolic pressure to increase by $18-90 \mathrm{mmHg}$ in the quadriplegics and by $15-25 \mathrm{mmHg}$ in the paraplegics (Brown et al., 2009b). Skin blood flow also fell during vibration, again representing an apparently generalized constriction of all blood vessels below lesion. Importantly, there was an inverse relationship between blood pressure and skin blood flow below the lesion, but a poor relationship between the clinical signs and the observed increase in blood pressure: some individuals reported severe headaches with reflex increases in systolic pressure of $35 \mathrm{mmHg}$, whereas others had no headache or other signs despite increases in systolic pressure exceeding $50 \mathrm{mmHg}$. For the sake of the current discussion, what is also important to recognize is that vibration of the penis is a non-noxious stimulus, one that generates marked somatosympathetic reflexes.

\section{SOMATOSYMPATHETIC REFLEXES TO NOXIOUS INPUTS IN HUMAN SPINAL CORD INJURY}

We recently showed that selective stimulation of muscle nociceptors, induced by bolus intramuscular injection of hypertonic saline into the leg of awake human subjects, caused a strong, dull ache that lasted $\sim 8 \mathrm{~min}$ and produced an increase in MSNA and modest increase in blood pressure and heart rate; these followed the time course of the subjective report of pain (Burton et al., 2009a). Conversely, SSNA showed only a transient increase, associated with a fall in skin blood flow, which we believe is simply a reflection of the alerting (arousal) response to the pain (Burton et al., 2009b). Interestingly, when similar injections were made into the legs of people with SCI (i.e., below the level of the lesion) the predicted increases in blood pressure and decreases in skin blood flow did not occur; in fact, there was a slight fall in blood pressure (Burton et al., 2008). Figure 2 compares the changes in cutaneous vasoconstriction, blood pressure, and heart rate to noxious stimulation (produced by intramuscular injection of hypertonic saline below lesion) and innocuous stimulation (produced by low-intensity electrical stimulation over the abdominal wall) in patients with SCI. It is also worth pointing out that the absence of any sustained cutaneous vasoconstriction to noxious stimulation argues against the idea that an ongoing noxious stimulus would, via a spinal

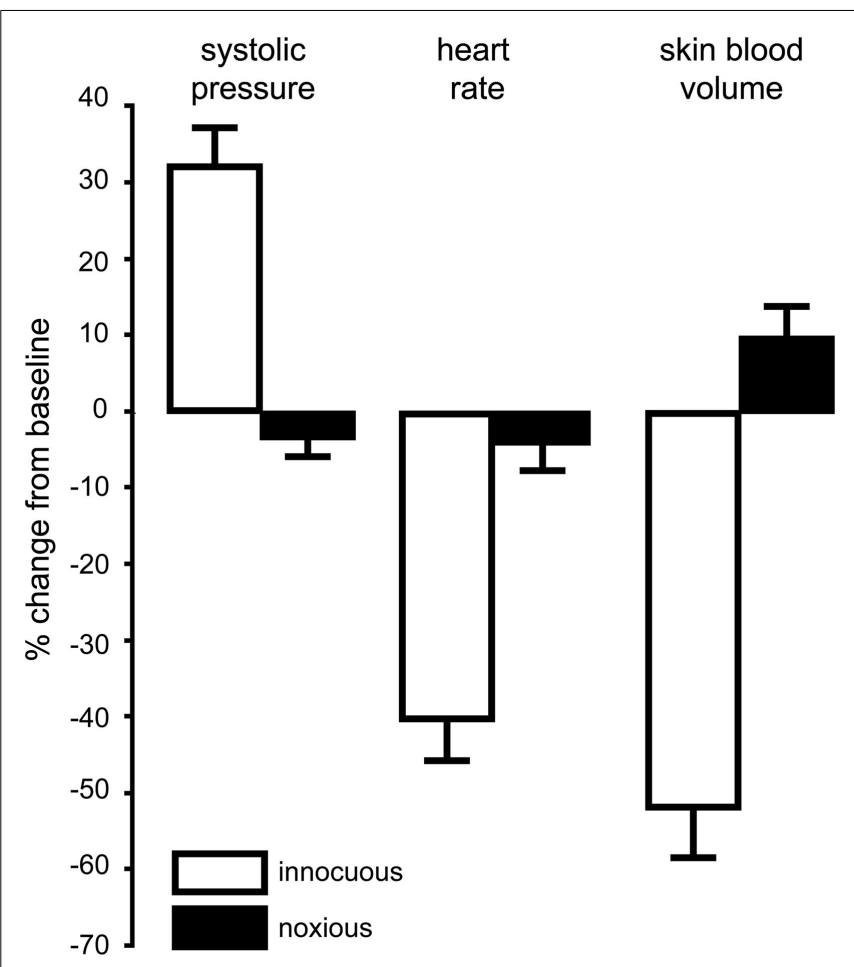

FIGURE 2 | Mean changes ( \pm SE) in blood pressure, heart rate, and cutaneous vasoconstriction in the toes during innocuous stimulation (electrical stimulation of the abdominal wall with a $1 \mathrm{~s} 20 \mathrm{~Hz}$ train; $n=12$ ) or noxious stimulation (intramuscular injection of $1 \mathrm{ml}$ hypertonic saline into tibialis anterior; $n=12$ ) below lesion in patients with spinal cord injury. Data combined from Brown et al. (2007) and Burton et al. (2008).

reflex, cause prolonged decreases in skin blood flow and thereby compromise wound healing.

Based on the above observations in able-bodied subjects, we now believe that the increase in sympathetic vasoconstrictor drive, and the resultant increase in blood pressure, evoked by noxious stimuli reflect part of the affective responses to pain, and are related to the emotional quality of the pain rather than to any spinal reflex. Indeed, brain imaging during intramuscular injection of hypertonic saline has revealed discrete activation of cortical areas involved in the affective responses to pain - the subgenual anterior cingulate and insular cortices - as well as the primary somatosensory cortex (Henderson et al., 2006). That selective stimulation of nociceptors in skin or muscle below lesion does not cause vasoconstriction or increases in blood pressure goes against existing dogma, in which autonomic dysreflexia is believed to be, in many cases, triggered by noxious inputs below the lesion (Karlsson, 1999). Indeed, here is a common definition found in the literature for the cause of autonomic dysreflexia: “. . any stimulus that might cause pain in a person without spinal cord injury is capable of triggering autonomic dysreflexia" (Blackmer, 2003; Selcuk et al., 2004). Moreover, stimuli that would undoubtedly be noxious in intact individuals often fail to induce autonomic dysreflexia in those with SCI. For example, although pressure ulcers may be quite painful in intact individuals (Roth et al., 2004), and 
there are reported cases of autonomic dysreflexia being associated with pressure sores (Teasell et al., 2000), clinical experience does not support the concept that most spinal patients who have pressure ulcers consistently and continually suffer from constant autonomic dysreflexia - the presence of an assumed continuous nociceptive stimulus does not necessarily translate into a continual hypertensive state. Another example from our clinical experience: a patient with T6 complete lesion and a known clinical history of autonomic dysreflexia experienced a spiral fracture of his right femur after a fall. Despite his predisposition to autonomic dysreflexia, regular observations of heart rate, and blood pressure were stable following the fall and he did not experience any symptoms of autonomic dysreflexia following the fracture.

\section{PLASTIC CHANGES IN THE SPINAL CORD FOLLOWING SPINAL CORD INJURY}

While SCI makes it easier to see spinal somatosympathetic reflexes in human subjects, are there changes in the spinal circuitry responsible for these reflexes following SCI? This is difficult to answer, because any reflexly generated increase in sympathetic vasoconstriction would be buffered by the baroreflex in intact individuals. We know that electrical stimulation of the abdominal wall (or anywhere on the body surface) in intact individuals causes cutaneous vasoconstriction and sweat release, but this does not mean it is spinally generated; conversely, the vasoconstrictor response to such stimulation in spinal patients can only be spinally mediated. Moreover, the fact that noxious stimulation below lesion - produced by intramuscular or subcutaneous injection of hypertonic saline - causes no cutaneous vasoconstriction, no sweat release, no increase in blood pressure, and no decrease in heart rate indicates that the increase in muscle and cutaneous sympathetic nerve activity produced by these stimuli in intact individuals is generated supraspinally. It is quite possible that the spinally mediated increase in sympathetic outflow to somatosensory stimulation following SCI is exaggerated: we know that the duration of the cutaneous vasoconstriction is increased in human SCI (Brown et al., 2007, 2009a), but this may well reflect an augmented peripheral - as opposed to central - response. Indeed, in the spinal rat blood vessels show an increased reactivity to direct stimulation of the vasomotor nerves (Yeoh et al., 2004a,b; Brock et al., 2006; McLachlan and Brock, 2006; Rummery et al., 2010). It is now believed that neurovascular transmission is potentiated by both an increase in neurotransmitter release and a reduction in noradrenaline reuptake, depending on the vessel (Brock et al., 2006; McLachlan, 2007; Rummery et al., 2010). So, while this can be

\section{REFERENCES}

Alexander, M. S., Biering-Sorensen, F., Bodner, D., Brackett, N. L., Cardenas, D., Charlifue, S., Creasey, G., Dietz, V., Ditunno, J., Donovan, W., Elliott, S. L., Estores, I., Graves, D. E., Green, B., Gousse, A., Jackson, A. B., Kennelly, M., Karlsson, A. K., Krassioukov, A., Krogh, K., Linsenmeyer, T., Marino, R., Mathias, C. J., Perkash, I., Sheel, A. W., Schilero, G., Schurch, B., Sonksen,

interpreted as a reflection of "peripheral plasticity" following SCI, we are still left with the question as to whether there are plastic changes centrally.

Studies conducted in experimental animals have demonstrated anatomical changes in preganglionic neurons following a SCI an initial loss but subsequent regrowth of synapses (Krassioukov and Weaver, 1995, 1996) - and plastic changes in the strength of the synaptic connections between preganglionic and postganglionic sympathetic neurons following section of the axons of preganglionic neurons: those neurons that remain exhibit extensive collateral sprouting (Murray and Thompson, 1957; Liestol et al., 1987). Moreover, by removing all but one set of segmental inputs to a paravertebral ganglion one can demonstrate plastic changes in synaptic strength over time: immediately following surgery strong synaptic inputs were present on only $10 \%$ of the postganglionic neurons, whereas after several weeks the number of these synapses had increased to some 70\% (Ireland, 1999). This indicates that the residual preganglionic neurons had sprouted and formed strong synaptic connections, even on neurons that had been completely deprived of synaptic drive by the lesion. Indeed, it has been argued that formation of aberrant strong inputs may contribute to the exaggerated vasoconstrictor responses to sensory inputs below lesion in SCI (McLachlan, 2007), and evidence from rats has shown that sprouting of small-diameter primary afferent neurons occurs within the spinal cord following a severe but not a mild compression injury of the midthoracic cord (Weaver et al., 2001). Moreover, the magnitude of the autonomic dysreflexia induced by colonic distension is related to the degree of primary afferent sprouting (Weaver et al., 2001), and dysreflexia can be abolished by blocking this sprouting (Krenz et al., 1999). It is highly likely that such plastic changes, both afferent and efferent, also occur within the spinal cord in human SCI.

\section{CONCLUSION}

We conclude that robust somatosympathetic reflexes can be produced by innocuous stimuli below lesion in human SCI, whereas specific activation of nociceptors below lesion does not generate such reflexes. Not withstanding the importance of viscerosympathetic reflexes, this argues against the existing clinical dogma that autonomic dysreflexia is often triggered by noxious somatic inputs originating below a spinal lesion. Moreover, the absence of sustained cutaneous vasoconstriction to noxious inputs originating below a spinal lesion argues against the idea that an ongoing noxious stimulus, such as that produced by a pressure ulcer, would reduce skin blood flow and potentially compromise wound healing.

Beacham, W. S., and Perl, E. R. (1964). Characteristics of a spinal sympathetic reflex. J. Physiol. Paris 173, 431-448.

Blackmer, J. (2003). Rehabilitation medicine: 1. Autonomic dysreflexia. Can. Med. Assoc. J. 169, 931-935.

Brackett, N. L., Ferrell, S. M., Aballa, T. C., Amador, M. J., Padron, O. F., Sonksen, J., and Lynne, C. M. (1998). An analysis of 653 trials of penile vibratory stimulation in men with spinal cord injury. J. Urol. 159, 1931-1934.

Brock, J. A., Yeoh, M., and McLachlan, E. M. (2006). Enhanced neurally evoked responses and inhibition of norepinephrine reuptake in rat mesenteric arteries after spinal transection. Am. J. Physiol. Heart Circ. Physiol. 290, H398-H405. 
Brown, R., Burton, A. R., and Macefield, V. G. (2009a). Input-output relationships of a somatosympathetic reflex in human spinal cord injury. Clin. Auton. Res. 19, 213-220.

Brown, R., Stolzenhein, G., Engel, S., and Macefield, V. G. (2009b). Cutaneous vasoconstriction as a measure of incipient autonomic dysreflexia during penile vibratory stimulation in spinal cord injury. Spinal Cord 47 , 538-544.

Brown, R., Engel, S., Wallin, B. G., Elam, M., and Macefield, V. G. (2007). Assessing the integrity of sympathetic pathways in spinal cord injury. Auton. Neurosci. 134, 61-68.

Brown, R., and Macefield, V. G. (2008). Assessing the capacity of the sympathetic nervous system to respond to a cardiovascular challenge in human spinal cord injury. Spinal Cord 46, 666-672.

Burton, A., Brown, R., and Macefield, V. G. (2008). Selective activation of muscle and skin nociceptors does not trigger exaggerated sympathetic responses in spinal injured subjects. Spinal Cord 46, 660-665.

Burton, A. R., Birznieks, I., Bolton, P. S., Henderson, L. A., and Macefield, V. G. (2009a). The effects of deep and superficial experimentally-induced acute pain on muscle sympathetic nerve activity in human subjects. $J$. Physiol. Paris 587, 183-193.

Burton, A. R., Birznieks, I., Spaak, J., Henderson, L. A., and Macefield, V. G. (2009b). The effects of deep and superficial experimentallyinduced acute pain on skin sympathetic nerve activity in human subjects. Exp. Brain Res. 195, 317-324.

Cariga, P., Catley, M., Mathias, C. J., Savic, G., Frankel, H. L., and Ellaway, P. H. (2002). Organization of the sympathetic skin response in spinal cord injury. J. Neurol. Neurosurg. Psychiatr. 72, 356-360.

Coote, J. H., and Downman, C. B. B. (1966). Central pathways of some autonomic reflex discharges. J. Physiol. Paris 183, 714-729.

Coote, J. H., Downman, C. B. B., and Weber, W. V. (1969). Reflex discharges into thoracic white rami elicited by somatic and visceral afferent excitation. J. Physiol. Paris 202, 147-159.

Delius, W., Hagbarth, K. E., Hongell, A., and Wallin, B. G. (1972a). Manoeuvres affecting sympathetic outflow in human muscle nerves. Acta Physiol. Scand. 84, 82-94.

Delius, W., Hagbarth, K. E., Hongell, A., and Wallin, B. G. (1972b). Manoeuvres affecting sympathetic outflow in human skin nerves. Acta Physiol. Scand. 84, 177-186.

Fagius, J., and Karhuvaara, S. (1989). Sympathetic activity and blood pressure increases with bladder distension in humans. Hypertension 14, 511-517.

Fuhrer, M. J. (1975). Effects of stimulus site on the pattern of skin conductance responses evoked from spinal man. J. Neurol. Neurosurg. Psychiatr. 38, 749-755.

Gimovski, M. L., Ojeda, A., Ozaki, R., and Zerne, S. (1985). Management of autonomic hyperreflexia associated with a low thoracic spinal cord lesion. Am. J. Obstet. Gynecol. 153, 223-224.

Henderson, L. A., Bandler, R., Gandevia, S. C., and Macefield, V. G. (2006). Distinct forebrain activity patterns during deep versus superficial pain. Pain 120, 286-296.

Horeyseck, G., and Jänig, W. (1974). Reflex activity in postganglionic fibres within skin and muscle nerves elicited by somatic stimuli in chronic spinal cats. Exp. Brain Res. 21, 155-168.

Ireland, D. R. (1999). Preferential formation of strong synapses during re-innervation of guinea-pig sympathetic ganglia. J. Physiol. Paris 520, 827-837.

Jänig, W., and Spilok, N. (1978). Functional organization of the sympathetic innervation supplying the hairless skin of the hindpaws in chronic spinal cats. Pflugers Arch. 377, 25-31.

Karlsson, A.-K. (1999). Autonomic dysreflexia. Spinal Cord 37, 383-291.

Karlsson, A.-K. (2006). Autonomic dysfunction in spinal cord injury: clinical presentation of symptoms and signs. Prog. Brain Res. 152, $1-8$.

Karlsson, A. K., Friberg, P., Lonnroth, P., Sullivan, L., and Elam, M. (1998). Regional sympathetic function in high spinal cord injury during mental stress and autonomic dysreflexia. Brain 121, 1711-1719.

Kerman, I. A., and Yates, B. J. (1999). Patterning of somatosympathetic reflexes. Am. J. Physiol. Regul. Integr. Comp. Physiol. 277, R716-R724.

Krassioukov, A. V., and Weaver, L. C. (1995). Reflex and morphological changes in spinal preganglionic neurons after cord injury in rats. Clin. Exp. Hypertens. 17, 361-373.

Krassioukov, A. V., and Weaver, L. C. (1996). Morphological changes in sympathetic preganglionic neurons after spinal cord injury in rats. $\mathrm{Neu}$ roscience 70, 211-226.
Krenz, N. R., Meakin, S. O., Krassioukov, A. V., and Weaver, L. C. (1999). Neutralizing intraspinal nerve growth factor blocks autonomic dysreflexia caused by spinal cord injury. J. Neurosci. 19, 7405-7414.

Liestol, K., Maehlen, J., and Nja, A. (1987). Two types of synaptic selectivity and their interrelation during sprouting in the guinea-pig superior cervical ganglion. J. Physiol. Paris 384, 233-245.

Lin, C. S., Macefield, V. G., Elam, M. Wallin, B. G., Engel, S., and Kiernan, M. C. (2007). Axonal changes in spinal cord injured patients distal to the site of injury. Brain 130, 985-994.

Linsenmeyer, T. A., Campagnolo, D. I., and Chou, I. H. (1996). Silent autonomic dysreflexia during voiding in men with spinal cord injuries. $J$. Urol. 155, 519-522.

Marino, R. J., Barros, T., BieringSorensen, F., Burns, S. P., Donovan, W. H., Graves, D. E., Haak, M., Hudson, L. M., and Priebe, M. M. (2003). International standards for neurological classification of spinal cord injury. $J$. Spinal Cord Med. 26(Suppl. 1) S50-S56.

Marsh, D. R., and Weaver, L. C. (2004) Autonomic dysreflexia, induced by noxious or innocuous stimulation, does not depend on changes in dorsal horn substance p. J. Neurotrauma 21, 817-828.

Mathias, C. J., and Frankel, H. L. (2002). "Autonomic disturbances in spinal cord lesions," in Autonomic Failure: A Textbook of Clinical Disorders of the Autonomic Nervous System, 4th Edn, eds C. J. Mathias and R. Bannister (New York: Oxford University Press), 494-513.

Mathias, C. J, Frankel, H. L., Christensen, N. J., and Spalding, J. N. K. (1976). Enhanced pressor response to noradrenaline in patients with cervical spinal cord transection. Brain 99, 757-770.

McLachlan, E. M. (2007). Diversity of sympathetic vasoconstrictor pathways and their plasticity after spinal cord injury. Clin. Auton. Res. 17, 6-12.

McLachlan, E. M., and Brock, J. A. (2006). Adaptations of peripheral vasoconstrictor pathways after spinal cord injury. Prog. Brain Res. 152, 289-297.

McMullan, S., Pathmanandavel, K., Pilowsky, P. M., and Goodchild, A. K. (2008). Somatic nerve stimulation evokes qualitatively different somatosympathetic responses in the cervical and splanchnic sympathetic nerves in the rat. Brain Res. 1217, 139-147.

Murray, J. G., and Thompson, J. W. (1957). The occurrence and function of collateral sprouting in the sympathetic nervous system of the cat. J. Physiol. Paris 135, 133-162.

Nicotra, A., Young, T. M., Asahina, M., and Mathias, C. J. (2005) The effect of different physiological stimuli on skin vasomotor reflexes above and below the lesion in human chronic spinal cord injury. Neurorehabil. Neural Repair 19, 325-331.

Ogura, T., Kubo, T., Lee, K., and Katayama, Y. (2004). Sympathetic skin responses in patients with spinal cord injury. J. Orthop. Surg. 12, 35-39.

Reitz, A., Schmid, D. M., Curt, A., Knapp, P. A., and Schurche, B. (2003). Autonomic dysreflexia in response to pudendal nerve stimulation. Spinal Cord 41, 539-542.

Roth, R. S., Lowery, J. C., and Hamill, J. B. (2004). Assessing persistent pain and its relation to affective distress, depressive symptoms, and pain catastrophizing in patients with chronic wounds: a pilot study. Am. J. Phys. Med. Rehab. 83, 827-834.

Rummery, N. M, Tripovic, D., McLachlan, E. M., and Brock, J. A. (2010). Sympathetic vasoconstriction is potentiated in arteries caudal but not rostral to a spinal cord transection in rats. J. Neurotrauma 27 , 2077-2089.

Saper, C. B., and DeMarchena, O. (1986). Somatosympathetic reflex unilateral sweating and pupillary dilatation in a paraplegic man. Ann. Neurol. 19, 389-390.

Sato, A., and Schmidt, R. F. (1971). Spinal and supraspinal components of the reflex discharges into lumbar and thoracic white rami. J. Physiol. Paris 212, 839-850.

Sato, A., and Schmidt, R. F. (1973). Somato-sympathetic reflexes: afferent fibres, central pathways, discharge characteristics. Physiol. Rev. 53, 916-947.

Selcuk, B., Inanir, M., Kurtaran, A., Sulubut, N., and Akyuz M. (2004). Autonomic dysreflexia after intramuscular injection in traumatic tetraplegia. Am. J. Phys. Med. Rehabil. 83, 61-64.

Stjernberg, L., Blumberg, H., and Wallin, B. G. (1986). Sympathetic activity in man after spinal cord 
injury. Outflow to muscle below the lesion. Brain 109, 695-715.

Teasell, R. W., Arnold, J. M. O., Krassioukov, A., and Delaney, G. A. (2000). Cardiovascular consequences of loss of supraspinal control of the sympathetic nervous system after spinal cord injury. Arch. Phys. Med. Rehabil. 81, 506-516.

Wallin, B. G., and Stjernberg, L. (1984). Sympathetic activity in man after spinal cord injury. Outflow to skin below the lesion. Brain 107, 183-198.

Weaver, L. C., Verghese, P., Bruce, J. C., Fehlings, M. G., Krenz, N. R., and Marsh, D. R. (2001). Autonomic dysreflexia, and primary afferent sprouting after clip-compression injury of the rat spinal cord. $\mathrm{J}$. Neurotrauma 18, 1107-1119.

Wieder, J. A., Brackett, N. L., Lynne, C. M., Green, J. T., and Aballa, T. C. (2000). Anesthetic block of the dorsal penile nerve inhibits vibratoryinduced ejaculation in men with spinal cord injuries. Urology 55, 915-917.

Yeoh, M., McLachlan, E. M., and Brock, J. A. (2004a). Tail arteries from chronically spinalized rats have potentiated responses to nerve stimulation in vitro. J. Physiol. Paris 556, 545-555.

Yeoh, M., McLachlan, E. M., and Brock, J. A. (2004b). Chronic decentralization potentiates neurovascular transmission in the isolated rat tail artery, mimicking the effects of spinal transection. J. Physiol. Paris 561, 583-596.

Conflict of Interest Statement: The authors declare that the research was conducted in the absence of any commercial or financial relationships that could be construed as a potential conflict of interest.

Received: 04 March 2012; paper pending published: 18 April 2012; accepted: 30 May 2012; published online: 25 June 2012.
Citation: Macefield VG, Burton AR and Brown $R$ (2012) Somatosympathetic vasoconstrictor reflexes in human spinal cord injury: responses to innocuous and noxious sensory stimulation below lesion. Front. Physio. 3:215. doi: 10.3389/fphys.2012.00215

This article was submitted to Frontiers in Integrative Physiology, a specialty of Frontiers in Physiology.

Copyright (C) 2012 Macefield, Burton and Brown. This is an open-access article distributed under the terms of the Creative Commons Attribution Non Commercial License, which permits noncommercial use, distribution, and reproduction in other forums, provided the original authors and source are credited. 